\title{
EXPORT: Near-IR observations of Vega-type and pre-main sequence stars ${ }^{\star}$
}

\author{
C. Eiroa ${ }^{1}$, F. Garzón ${ }^{2}$, A. Alberdi ${ }^{3}$, D. de Winter ${ }^{4}$, R. Ferlet ${ }^{5}$, C. A. Grady ${ }^{6}$, A. Cameron ${ }^{7}$, J. K. Davies ${ }^{8}$,
} H. J. Deeg ${ }^{3}$, A. W. Harris ${ }^{9}$, K. Horne ${ }^{7}$, B. Merín ${ }^{10}$, L. F. Miranda ${ }^{3}$, B. Montesinos ${ }^{10}$, A. Mora ${ }^{1}$, R. Oudmaijer ${ }^{11}$, J. Palacios ${ }^{1}$, A. Penny ${ }^{12}$, A. Quirrenbach ${ }^{13}$, H. Rauer ${ }^{9}$, J. Schneider ${ }^{14}$, E. Solano ${ }^{10}$, Y. Tsapras ${ }^{7}$, and P. R. Wesselius ${ }^{15}$

1 Dpto. Física Teórica, C-XI, Facultad de Ciencias, Universidad Autónoma de Madrid, Cantoblanco, 28049 Madrid, Spain

2 Instituto de Astrofísica de Canarias, 38200 La Laguna, Tenerife, Spain

3 Instituto de Astrofísica de Andalucía, Apartado de Correos 3004, 18080 Granada, Spain

4 TNO/TPD-Space Instrumentation, Stieltjesweg 1, PO Box 155, 2600 AD Delft, The Netherlands

${ }^{5}$ CNRS, Institute d'Astrophysique de Paris, 98bis Bd. Arago, 75014 Paris, France

6 NOAO/STIS, Goddard Space Flight Center, Code 681, NASA/GSFC, Greenbelt, MD 20771, USA

7 Physics \& Astronomy, University of St. Andrews, North Haugh, St. Andrews KY16 9SS, Scotland, UK

8 Joint Astronomy Centre, 660 N. A'ohoku Place, Hilo, Hawaii 96720, USA

9 DLR Department of Planetary Exploration, Rutherfordstrasse 2, 12489 Berlin, Germany

10 L.A.E.F.F., VILSPA, Apartado de Correos 50727, 28080 Madrid, Spain

11 Department of Physics and Astronomy, University of Leeds, Leeds LS2 9JT, UK

12 Rutherford Appleton Laboratory, Didcot, Oxfordshire OX11 0QX, UK

13 Department of Physics, Center for Astrophysics and Space Sciences, University of California San Diego, Mail Code 0424, La Jolla, CA 92093-0424, USA

14 Observatoire de Paris, 92195 Meudon, France

15 SRON, Universiteitscomplex "Zernike", Landleven 12, PO Box 800, 9700 AV Groningen, The Netherlands

Received 18 August 2000 / Accepted 12 October 2000

\begin{abstract}
We present near-IR $J H K$ photometric data of a sample of 58 main-sequence, mainly Vega-type, and pre-main sequence stars. The data were taken during four observing runs in the period May 1998 to January 1999 and form part of a coordinated effort with simultaneous optical spectroscopy and photo-polarimetry. The near-IR colors of the MS stars correspond in most cases to photospheric colors, although noticeable reddening is present towards a few objects, and these stars show no brightness variability within the observational errors. On the other hand, the PMS stars show near-IR excesses and variability consistent with previous data.
\end{abstract}

Key words. stars: emission-line, Be - stars: pre-main sequence - stars: variable: general - infrared: stars

\section{Introduction}

The discovery of far-IR excesses associated with Vega, $\beta$ Pic and some other similar stars was one of the major scientific outcomes of the IRAS mission. These findings indicate the presence of significant amounts of solid material around main-sequence stars other than the Sun (e.g. Backman \& Paresce 1993). Coronographic observations

Send offprint requests to: C. Eiroa,

e-mail: carlos@xiada.ft.uam.es

* Tables 1 and 2 are only available in electronic form at the CDS via anonymous ftp to cdsarc.u-strasbg.fr

(130.79.128.5) or via

http://cdsweb.u-strastg.fr/cgi-bin/qcat?J/A+A/365/110 revealed that the dust in $\beta$ Pic is distributed in an edgeon disk (Smith \& Terrile 1984). Transient spectroscopic events in the lines of many UV and optical lines have been interpreted as being due to infalling comet-like bodies, while asymmetries in the disk have been attributed to the presence of a planet in the $\beta$ Pic system (see Lecavelier des Etangs 2000, for a recent review on $\beta$ Pic). Thus, it seems to be beyond doubt that the young (age $t \sim 10^{7}$ years, Barrado y Navascués et al. 1999), main-sequence star $\beta$ Pic harbors a protoplanetary disk in which planets are being formed, and perhaps have already formed. In this respect it should be noted that the G8 V star $\rho^{1}$ Cnc, with an age of $t \sim 510^{9} \mathrm{yr}$ and a Jupiter-like planet orbiting it, also shows the presence of a Vega-like dust disk 
in data from ISO (Dominik et al. 1998). On the other side of the stellar evolutionary path, it has been suggested on the basis of various observational data that a population of pre-main sequence stars may be $\beta$ Pic progenitors (Grady et al. 2000). Thus, an observational framework tracing the path of the formation of planetary systems, from protostellar disks to well developed planets, is growing.

The 1998 international observing time of the Canary Islands Observatories was allocated to the EXPORT collaboration (EXoPlanetary Observational Research Team) and a considerable fraction of this time was dedicated to observations of a large sample of main sequence, mainly Vega-type stars, and pre-main sequence stars (Eiroa et al. 2000). The aim of this effort was to study stars at different evolutionary stages to provide some clues regarding protoplanetary disk evolution. The main EXPORT observations consisted of intermediate and high resolution optical spectra supported by simultaneous optical photopolarimetry and near-IR photometry. The stars in this sample were monitored over a range of different timescales from days to months. In this paper we present the near-IR data we collected and a brief description of the general near-IR behavior of the sample. Detailed analysis of these results in combination with the rest of the EXPORT observations will be presented elsewhere.

\section{Observations}

Near-IR data were obtained at the Teide Observatory (Tenerife, Canary Islands) with the $1.5 \mathrm{~m}$ Carlos Sánchez Telescope (CST) during four observing runs: 1998 14-17 May, 28-31 July, 23-26 October and 1999 28-31 January. There were a total of 13 useful (some only partially) nights. We used two different instrumental setups throughout the runs. A single channel NIR photometer equipped with an InSb detector was used in May and October; a 15 arcsec beam and a chopper-throw of 30 arcsec were selected for these observations. During the July and January runs CAIN, a 2D NIR camera equipped with a NICMOS $256 \times 256 \mathrm{HgCdTe}$ array, was used. The photometer was operated in standard AC mode, chopping between two adjacent beams, with no further adjustments. The images taken with CAIN were flat fielded and dark current corrected using standard procedures. A minimum of two different frame series were taken for each object, shifting the targets throughout the detector quadrants to remove systematic effects. The averaged series in different quadrants were then subtracted one from another to remove the sky contribution to the measured signal. The time interval between two adjacent series was kept below 2 minutes to avoid background variations of the atmospheric emission. Due to the brightness of some sources $(K<8 \mathrm{mag})$ we defocused the camera in order to avoid departures from the linear range of the NICMOS array. Errors introduced by the defocusing, together with sky variations, are well below $0.1 \mathrm{mag}$, as estimated from the sigmavalue of the averaged results. Observations with the single channel photometer and CAIN were carried out through standard broad-band JHK filters. Cross-correlations between measurements taken with both instruments of the same objects show negligible systematic deviations and no filter correction has been applied to the results. Data reduction proceeded in a standard way. Magnitude errors estimated from a least-squares fit to the standard star data are $\leq 0.02 \mathrm{mag}(1 \sigma \mathrm{rms})$ in each filter for the data taken with the single-detector photometer. Errors are larger, around $0.05 \mathrm{mag}(1 \sigma \mathrm{rms})$, for the data taken with the camera.

\section{Results}

The observed sample consists of 58 objects: 40 are PMS stars (HAeBe and T Tauri stars) and 18 MS stars (Vegatype and Lindroos post-T Tauri systems). The stars were observed once per night whenever they were observable from the Canary Islands; in a few cases there are several observations per night. Thus we obtained daily (approx. 24 hours) and 3-monthly (approx. 90 days) monitoring for most of the stars.

Tables 1 and 2 summarize the results of the near-IR observations for the MS stars and the PMS objects respectively. In general, we consider objects variable when different measurements in each filter differ by more than the conservative limit of $5 \sigma$, i.e. 0.1 magnitudes in the May 98 and October 98 observing runs. For the July 98 and January 99 runs, we take the value of 0.15 magnitudes, i.e. $3 \sigma$, but also taking into account the results of the other two observing periods.

\section{MS stars}

This group of stars includes double systems in which the secondary has been classified as a post-T Tauri (PTT) star, while the primary is an early type star (Lindroos 1986); in some cases they show far-infrared excesses revealing the presence of cool dust in the circumstellar environment (Ray et al. 1995). Only in one case of the PTT systems, HR 4757, were our near-IR observations carried out for both components of the double; for the rest we observed the brighter component. All these stars are referred to as PTT in Table 1. In addition, we include in this group A-shell stars and other stars which show properties similar to Vega and $\beta$ Pic, i.e. IRAS excess and/or optical evidence of circumstellar gas (Backman \& Paresce 1993; Stencel \& Backman 1991; Cheng et al. 1991; LagrangeHenri et al. 1990). These stars are also referred to as "Vega" in Table 1.

Seven stars in our sample - HR 10, HR 26, HR 419, HR 836, HR 1369, HR 5422, and HR 9043 - have no previous published $J H K$ magnitudes. In the case of $\lambda$ Boo only the $K$ magnitude has previously been reported; in all other cases, our near-IR magnitudes are similar to those published previously. Table 3 compares our mean near-IR magnitudes with those found in the literature and it is apparent that there are no significant differences. All stars of this group are constant in brightness. The apparent variability of $\mathrm{BD}+31^{\circ} 643$ during the October 98 observations, 
Table 3. Comparison of the EXPORT magnitudes of the MS stars with other published results

\begin{tabular}{|c|c|c|c|c|}
\hline Object & $J$ & $H$ & $K$ & Reference \\
\hline \multirow[t]{2}{*}{49 Cet } & 5.51 & 5.51 & 5.51 & 7 \\
\hline & 5.54 & 5.51 & 5.51 & 2 \\
\hline \multirow{2}{*}{$\mathrm{BD}+31^{\circ} 643$} & 6.93 & 6.71 & 6.61 & 1 \\
\hline & & 6.69 & 6.47 & 3 \\
\hline \multirow[t]{2}{*}{ HD 23362} & 4.87 & 4.04 & 3.83 & 1 \\
\hline & 4.89 & 4.05 & 3.85 & 2 \\
\hline \multirow[t]{2}{*}{ HD 23680} & 6.02 & 5.38 & 5.24 & 1 \\
\hline & 6.01 & 5.37 & 5.24 & 2 \\
\hline \multirow[t]{2}{*}{ HD 34700} & 8.00 & 7.65 & 7.43 & 1 \\
\hline & 7.93 & 7.71 & 7.67 & 4 \\
\hline \multirow[t]{3}{*}{ HR 2174} & 5.44 & 5.39 & 5.37 & 1 \\
\hline & & 5.38 & 5.35 & 5 \\
\hline & & & 5.44 & 6 \\
\hline \multirow[t]{4}{*}{ HR 4757} & 2.99 & 3.01 & 3.07 & 1 \\
\hline & 3.06 & 3.08 & 3.05 & 7 \\
\hline & 3.01 & 3.03 & 3.03 & 8 \\
\hline & 3.04 & 3.08 & 3.07 & 9 \\
\hline \multirow[t]{2}{*}{ HR 4757B } & 6.77 & 6.34 & 6.25 & 1 \\
\hline & 6.81 & 6.38 & 6.27 & 7 \\
\hline \multirow[t]{2}{*}{$\lambda$ Boo } & 3.95 & 3.92 & 3.90 & 1 \\
\hline & & & 3.73 & 10 \\
\hline \multirow[t]{2}{*}{ HD 142764} & 6.35 & 5.55 & 5.34 & 1 \\
\hline & 6.41 & 5.58 & 5.35 & 2 \\
\hline \multirow[t]{2}{*}{ HD 218396} & 5.35 & 5.23 & 5.22 & 1 \\
\hline & 5.46 & 5.30 & 5.28 & 2 \\
\hline
\end{tabular}

(1) This work; (2) Sylvester et al. (1996); (3) Strom et al. (1974); (4) Coulson et al. (1998); (5) Allen (1973); (6) FajardoAcosta et al. (1998); (7) Glass (1974); (8) Lindroos (1983); (9) Bouchet et al. (1991); (10) McAlary \& McLaren (1980).

although it was the best period in terms of photometric quality, needs to be confirmed and we do not discuss it further.

Figure 1 presents the mean observed near-IR colors in a two color $(J-H, H-K)$ diagram of the 18 Vega-type and PTT stars in our sample. Of these, 13 stars have observed colors close to the intrinsic colors of their respective spectral types without any need for corrections due to interstellar or circumstellar extinction. Thus, the near-IR colors do not show any sign of the presence of hot circumstellar dust. These stars are also unpolarized in the optical (Oudmaijer et al. 2001). The rest behaves as follows.

HD 142764: The observed colors are compatible with a K5 V spectral type suffering considerable reddening. Assuming a normal interstellar extinction law (e.g. Rieke \& Lebofsky 1985) we estimate an optical extinction of $A_{v}$ $=1.8$, from the $J-H$ color. This is consistent with the extinction value deduced from optical photometry. The star also shows considerable polarization at these wavelengths (Oudmaijer et al. 2001).

BD $+31^{\circ} \mathbf{6 4 3}$ : This star's colors are compatible with a B5V star suffering a total extinction of $A_{v}=2.6$ magnitudes (from the $J-H$ color), in good agreement with the estimate of $A_{v}=2.8$ based on optical measurements (Snow et al. 1994; Oudmaijer et al. 2001). The star is

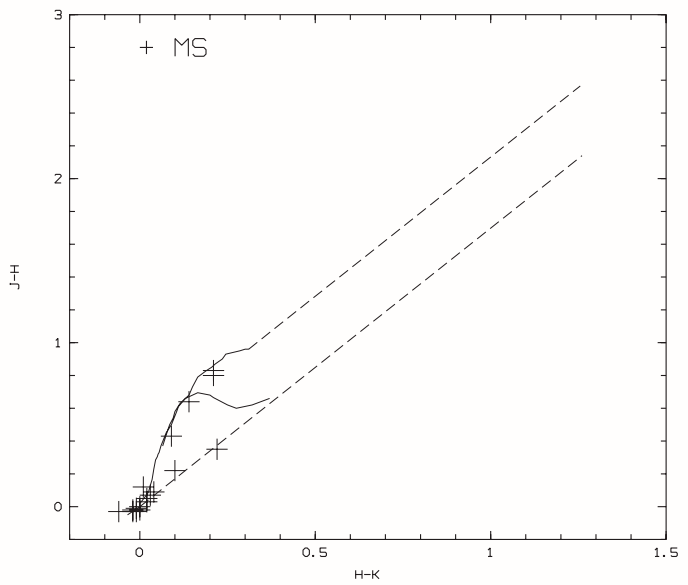

Fig. 1. Two Color diagram of EXPORT PTT and Vega-type stars

polarized in the optical (Andersson \& Wannier 1997; Oudmaijer et al. 2001); BD $+31^{\circ} 643$ has a dust disk which appears similar to that of $\beta$ Pic (Kalas \& Jewitt 1997).

HD 23362: Its colors are compatible with a K2 star suffering an extinction of $3 \mathrm{mag}$ (from the $J-H$ color), close to the reddening estimate of Sylvester et al. (1996); the star is polarized (Oudmaijer et al. 2001). Based on the optical spectrum, Dunkin et al. (1997) suggested that HD 23362 is not a young main-sequence star.

HD 23680: The $J-H$ color indicates an extinction of $A_{v}=2.5 \mathrm{mag}$, which is close to the extinction estimate from the $B-V$ color; the star is polarized in the optical (Oudmaijer et al. 2001).

HD 34700: The $J-H$ color is compatible with a G0V star with negligible extinction, in agreement with the optical photometry (Oudmaijer et al. 2001). However, the $H-K$ color seems to indicate a $H-K$ excess. This behavior of practically photospheric $J-H$ color but $H-K$ excess is also present in $51 \mathrm{Oph}$ (Waters et al. 1988). The $H-K$ excess seen in some Vega-type stars is probably due to non-photospheric flux contributing to the $K$ magnitude (Sylvester et al. 1996). HD 34700 also shows a low level of optical polarization (Oudmaijer et al. 2001).

\section{PMS stars}

\subsection{Color-color diagram}

Figure 2 shows the mean observed near-IR colors in a $(J-H, H-K)$ diagram. 35 out of the 40 PMS objects are clearly located to the right of the reddening line corresponding to an A0V star and show, therefore, a near-IR excess. Of the remaining 5 stars LKH $\alpha 200$ and $\mathrm{LKH} \alpha 262$ seem to have small near-IR excesses based on their late spectral types, K1 and M0 respectively (Cohen \& Kuhi 1979). To our knowledge this paper presents the first near-IR measurements of HD 158352 and HD 199143. Both stars have colors corresponding to a MS star of the 
Table 4. Observed source variations

\begin{tabular}{llrrrrrr}
\hline Object & Sp. T. & $\bar{J}$ & $\delta J$ & $\bar{H}$ & $\delta H$ & $\bar{K}$ & $\delta K$ \\
\hline VX Cas & A0 & 10.30 & 0.71 & 9.17 & 0.26 & 8.18 & 0.24 \\
LkH $\alpha$ 262 & K? & 10.84 & 0.56 & 10.07 & 0.62 & 9.71 & 0.52 \\
XY Per & B6 & 7.73 & 0.32 & 6.93 & 0.26 & 6.06 & 0.26 \\
CW Tau & K3 & 9.46 & 0.81 & 8.09 & 0.64 & 6.89 & 0.50 \\
RY Tau & G2/K1 & 7.35 & 0.32 & 6.31 & 0.22 & 5.46 & 0.21 \\
DK Tau & K7 & 8.89 & 0.40 & 7.88 & 0.37 & 7.10 & 0.36 \\
DR Tau & K4 & 8.94 & 0.40 & 7.84 & 0.39 & 6.91 & 0.36 \\
UX Ori & A2 & 8.88 & 0.19 & 8.24 & 0.33 & 7.41 & 0.36 \\
CO Ori & F9 & 8.71 & 0.65 & 7.72 & 0.49 & 6.81 & 0.35 \\
RY Ori & F6 & 9.93 & 1.10 & 9.17 & 0.90 & 8.51 & 0.80 \\
CQ Tau & F2 & 8.01 & 0.53 & 7.17 & 0.21 & 6.31 & 0.19 \\
T Ori & A3 & 8.42 & 0.27 & 7.39 & 0.11 & 6.37 & 0.11 \\
BF Ori & A6 & 9.00 & 0.15 & 8.48 & 0.12 & 7.80 & 0.22 \\
V350 Ori & A5 & 10.07 & 0.35 & 9.19 & 0.16 & 8.37 & 0.33 \\
VY Mon & B9 & 8.48 & 0.42 & 6.88 & 0.27 & 5.42 & 0.17 \\
KK Oph & A5V & 9.09 & 0.81 & 7.36 & 0.45 & 5.91 & 0.35 \\
HD 163296 & A3V & 6.29 & 0.32 & 5.59 & 0.22 & 4.79 & 0.16 \\
VV Ser & B3 & 8.84 & 0.36 & 7.57 & 0.27 & 6.42 & 0.27 \\
WW Vul & A0 & 9.24 & 0.21 & 8.33 & 0.23 & 7.41 & 0.25 \\
BM And & K5V & 9.98 & 0.38 & 9.09 & 0.21 & 8.44 & 0.19 \\
\hline
\end{tabular}

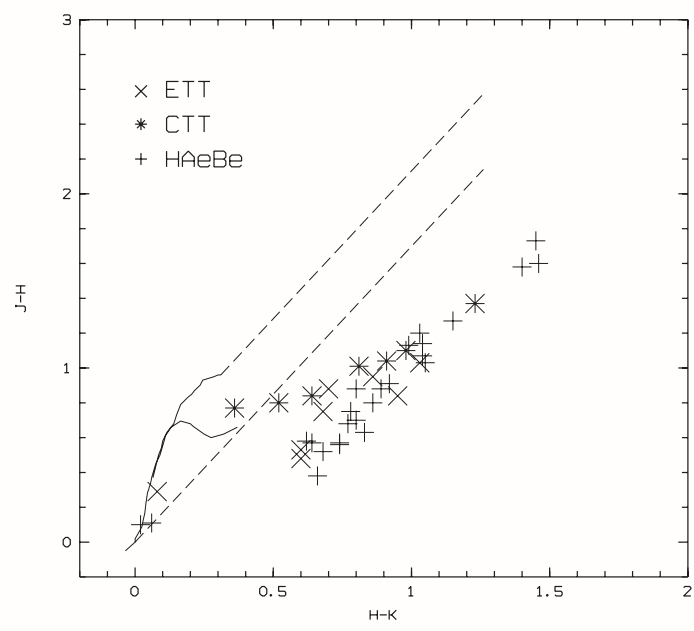

Fig. 2. Two color diagram of EXPORT PMS objects

same spectral type. The stars are unpolarized and the optical photometry is also consistent with their spectral type (Oudmaijer et al. 2001); it is likely that they have already reached the zero age main sequence. HD 141569 has nearIR colors roughly consistent with its spectral type, though with a small amount of reddening, $A_{V} \leq 1 \mathrm{mag}$ (the same is approximately true for the optical colors). Our magnitudes are brighter $(0.2-0.3 \mathrm{mag})$ than previous measurements (Malfait et al. 1998; Sylvester et al. 1996) but this is probably due to scattered light from the bright companions in its proximity (Pirzkal et al. 1997). The star is slightly polarized (Oudmaijer et al. 2001, see also Yudin \& Evans 1998). Interestingly, HD 141569 has a $\beta$ Pic disk (Augereau et al. 1999).
In Fig. 2 we plot the observed PMS stars in three groups: Classical T Tauri stars (CTTs) - spectral type G2 and later, early-type T Tauri stars (ETTs) - spectral types between F0 and G2, and Herbig Ae and Be stars spectral types earlier than F0. The position of the stars in the two-color diagram indicates that they occupy a region of the diagram characterized by different amounts of extinction and an excess with respect to the emission of a normal photosphere. The distributions of ETTs and HAeBe in the diagram differ slightly from that of CTTs. In addition, HAeBe and ETTs are located in a band displaced from the reddening band for main sequence stars, but more or less parallel to it. These characteristics are already known from previous work (e.g. Lada \& Adams 1992). These authors have shown that standard circumstellar disks can account for the near-IR colors of most $\mathrm{T}$ Tauri stars, but those models fail in the case of HAeBe stars. In the latter case, dust disks with central holes are required if self-luminous disks are the cause of the near-IR excess. The models do not provide a unique interpretation of near-IR colors in PMS stars, but they do indicate differences between low- and intermediate-mass young objects.

\subsection{Variability}

Twenty stars in our sample are variable on timescales of days and/or months. In some cases our monitoring is insufficient to establish if the objects have a periodic variability, although it is clear that in several cases the variability is irregular. Irregular variability is often observed in objects similar to those in our sample. Table 4 gives details of the stars in which we detect variability. A comparison with the literature (e.g. the SIMBAD data base) indicates, however, that some of the stars which remained 
constant during our observations do vary. In general, the variability observed in $J H K$ is roughly consistent with that observed at optical wavelengths (Oudmaijer et al. $2001)$, in the sense that all objects which are variable in the IR also vary at optical wavelengths.

In $\approx 50 \%$ of the sample stars the variability amplitude diminishes with the wavelength; no clear trend appears for the rest. The range of the amplitude is less than $0.4 \mathrm{mag}$ for most of the stars and goes down to our $3 \sigma$ limit. For 7 stars the variability is larger than 0.5 magnitudes in at least one filter, and in 3 out of the 7 (RY Ori, $\mathrm{LkH} \alpha$ 262 and CW Tau) that level of variability is found in all near-IR bands.

The origin of PMS variability is normally attributed to one or more of the the following causes (e.g. Herbst et al. 1994; Skrutskie et al. 1996; Herbst \& Shevchenko 1999): 1) cool stellar spots which rotationally modulate the star light, 2) hot spots caused by a magnetically channeled accretion flow onto the stellar surface, 3) variable obscuration by dust clumps in the circumstellar environment, 4) changes in the temperature profile and structure of the circumstellar dust disk, 5) stellar pulsations (Marconi \& Palla 1998). The first three causes can produce large amplitude variations, up to several magnitudes in the optical, with the amplitude diminishing with increasing wavelength. Variations in the temperature profile of the disk would produce more variability in the nearIR than in the optical since typical disk temperatures $\left(T_{\mathrm{D}}(\max ) \sim 2000 \mathrm{~K}\right)$ do not give rise to significant emission at visible wavelengths. Stellar pulsations should reveal themselves as periodic small-amplitude variations in the photometric behavior of the PMS stars. Thus, simultaneous observations at near-IR and optical wavelengths provide clues about the origin of the variability and which mechanisms act in the different wavelength ranges. A detailed analysis of the variability, considering the simultaneous near-IR and optical photo-polarimetric behavior, is beyond the scope of this paper and will be presented elsewhere.

\section{Conclusions}

We have presented the near-IR magnitudes of a sample of 58 main-sequence and pre-main sequence stars taken during the 1998 international time of the Canary Islands Observatories. For some of the stars in the sample these are the first published near-IR data. Although these data were taken in support of simultaneous optical spectroscopy, they reveal interesting aspects in their own right, such as reddening towards some Vega-type stars or the variability of some younger objects.

Acknowledgements. A. Alberdi, C. Eiroa, B. Merín, B. Montesinos, A. Mora, and J. Palacios are supported in part by Spanish grant ESP 98-1339

\section{References}

Allen, D. A. 1973, MNRAS, 161, 145

Anderson, B.-G., \& Wannier, P. G. 1997, ApJ, 491, 103

Augereau, J. C., Lagrange, A. M., Mouillet, D., \& Ménard, F. 1999, A\&A, 350, L51

Backman, D. E., \& Paresce, F. 1993, in Protostars and Planets III, ed. E. H. Levy, \& J. I. Lunine (Tucson: University of Arizona Press), 1253

Barrado y Navascués, D., Stauffer, J. R., Song, I., \& Caillault, J.-P. 1999, ApJ, 520, L123

Bouchet, P., Schmider, F. X., \& Manfroid, J. 1991, A\&AS, 91, 409

Cheng, K.-W., Grady, C. A., \& Bruhweiler, F. C. 1991, ApJ, $366, \mathrm{~L} 87$

Cohen, M., \& Kuhi, L. V. 1979, ApJS, 41, 743

Coulson, I. M., Walther, D. M., \& Dent, W. R. F. 1998, MNRAS, 296, 934

Dominik, C., Laureijs, R. J., Jourdain de Muizon, M., \& Habing, H. J. 1998, A\&A, 329, L53

Dunkin, S. K., Barlow, M. J., \& Ryan, S. G. 1997, MNRAS, 290,165

Eiroa, C., et al. (EXPORT) 2000, in Disks, Planetesimals and Planets, ed. F. Garzón, C. Eiroa, D. de Winter, \& T. Mahoney, ASP Conf. Ser., in press

Fajardo-Acosta, S. B., Telesco, C. M., \& Knacke, R. F. 1998, AJ, 115, 2101

Glass, I. S. 1974, MNSSA, 33, 53

Grady, C. A., Sitko, M. L., Russel, R. W., et al. 2000, in Protostars and Planets IV, ed. V. Mannings, A. P. Boss, \& S. S. Russell (Tucson: University of Arizona Press), 613

Herbst, W., Herbst, D. K., Grossman, E. J., \& Weinstein, D. 1994, AJ, 108, 1096

Herbst, W., \& Shevchenko, V. S. 1999, AJ, 118, 1043

Kalas, P, \& Jewitt, D. 1997, Nat, 386, 52

Lada, C. J., \& Adams, F. C. 1992, ApJ, 393, 278

Lagrange-Henri, A. M., Ferlet, R., Vidal-Madjar, A., et al. 1990, A\&AS, 85, 1089

Lecavelier des Etangs, A. 2000, in Disks, Planetesimals and Planets, ed. F. Garzón, C. Eiroa, D. de Winter, \& T. Mahoney, ASP Conf. Ser., in press

Lindroos, K. P. 1983, A\&AS, 51, 161

Lindroos, K. P. 1986, A\&A, 156, 223

Malfait, K., Bogaert, E., \& Waelkens, C. 1998, 331, 211

Marconi, M., \& Palla, F. 1998, ApJ, 507, L141

McAlary, C. W., \& McLaren, R. A. 1980, ApJ, 240, 853

Oudmaijer, R. D., et al. (EXPORT) 2001, A\&A, to be submitted

Pirzkal, N., Spillar, E. J., \& Dyck, H. M. 1997, ApJ, 481, 392

Ray, T. P., Sargent, A. I., Beckwith, S. V. M., Koresko, C., \& Kelly, P. 1995, ApJ, 440, L89

Rieke, G. H., \& Lebofsky, M. J. 1985, ApJ, 288, 618

Skrutskie, M. F., Meyer, M. R., Whalen, D., \& Hamilton, C. 1996, AJ, 112, 2168

Smith, B. A., \& Terrile, R. J. 1984, Sci, 226, 1421

Stencel, R. E., \& Backman, D. E. 1991, ApJS, 75, 905

Strom, S. E., Strom, K. A., \& Carrasco, L. 1974, PASP, 86, 798

Sylvester, R. J., Skinner, C. J., Barlow, M. J., \& Mannings, V. 1996, MNRAS, 279, 915

Waters, L. B. F. M., Coté, J., \& Geballe, T. R. 1988, A\&A, 203, 348

Yudin, R. V., \& Evans, A. 1998, A\&A, 131, 401 